\title{
Estabilidade e adaptabilidade do algodoeiro herbáceo no Cerrado do Mato Grosso e Mato Grosso do Sul
}

\author{
Adalgisa Aranha de Souza(1), Eleusio Curvelo Freire(2), Riselane de Lucena Alcântara Bruno(3) \\ Luiz Paulo de Carvalho ${ }^{(2)}$, João Luis da Silva Filho(2) e Walter Esfrain Pereira ${ }^{(3)}$
}

\begin{abstract}
(1)Escola Agrotécnica da Universidade Federal de Roraima, Campus Cauamé, BR 174, Monte Cristo, Km 12, CEP $69300-000$ Boa Vista, RR. E-mail: adaaranha@yahoo.com.br (2)Embrapa Algodão, Caixa Postal 174, CEP 58107-720 Campina Grande, PB. E-mail: eleusiofreire@hotmail.com, chpd@cnpa.embrapa.br, jlsilvafilho@uol.com.br (3)Universidade Federal da Paraíba, Centro de Ciências Agrárias, CEP 58397-000 Areia, PB. E-mail: lane@cca.ufpb.br, wep@cca.ufpb.br
\end{abstract}

\begin{abstract}
Resumo - O objetivo deste trabalho foi analisar a estabilidade e a adaptabilidade, e agrupar ambientes de cultivo, com dados de produtividade de oito genótipos do algodoeiro, avaliados em 16 ambientes localizados no Cerrado de Mato Grosso e Mato Grosso do Sul, na safra 1998/1999. O delineamento utilizado foi em quadrado latino 8x8. As análises individual e conjunta foram realizadas, considerando-se os efeitos de genótipos e ambientes fixos. Os parâmetros foram avaliados pelo método da ecovalência e pelo modelo AMMI. A linhagem CNPA 94773 foi a mais estável e adaptada às condições ambientais. Houve adaptabilidade específica e interação positiva de genótipos a grupos de ambientes: ITA 90 (Campo Novo dos Parecis, Chapadão do Sul, Sorriso, Itiquira e Sapezal); ITA 96, FMT Fetagri e FMT Saturno (Rondonópolis e Pedra Preta); BRS 197 (Nova Mutum, Primavera do Leste e Campo Verde); BRS FACUAL (Quatro Marcos e Campo Verde); CNPA 94773 (Ponta Porã, Sapezal, Alto Taquari e Lucas do Rio Verde) e BRS Antares (Ponta Porã, Alto Taquari e Lucas do Rio Verde).
\end{abstract}

Termos para indexação: Gossypium hirsutum, interação GxE, ecovalência, AMMI.

\section{Stability and adaptability of herbaceum cotton in the Cerrado of Mato Grosso and Mato Grosso do Sul, Brazil}

\begin{abstract}
The objective of this work was to analyze stability and adaptability, and to group environments with productivity data of eight cotton genotypes in 16 environments of Cerrado of Mato Grosso and Mato Grosso do Sul, Brazil, in 1998/1999 harvest. Statistical model used was the simple square 8x8. The individual and combined statistical analysis were performed by considering genotype and environment effects fixed. Parameters of the cultivars or lines were evaluated by the covalence, method and by the AMMI procedure. The lineage CNPA 94773 was the stablest and most adapted to environmental conditions. There was a positive specific adaptability and interaction of the genotypes with the environments: CNPA ITA 90 cultivar (Campo Novo dos Parecis, Chapadão do Sul, Sorriso, Itiquira and Sapezal); ITA 96, FMT Fetagri and FMT Saturno (Rondonópolis and Pedra Preta); BRS 197 (Nova Mutum, Primavera do Leste and Campo Verde); BRS FACUAL (Quatro Marcos and Campo Verde); CNPA 94773 (Ponta Porã, Sapezal, Alto Taquari and Lucas do Rio Verde) and BRS Antares (Ponta Porã, Alto Taquari and Lucas do Rio Verde).
\end{abstract}

Index terms: Gossypium hirsutum, GxE interaction, ecovalence, AMMI.

\section{Introdução}

A diversidade de ambientes para cultivo de algodoeiro no Cerrado leva a mudanças no comportamento de cultivares de algodão em diferentes áreas produtoras. Comportamento diferencial de uma cultivar em ambientes é o que se denomina interação genótipos $\mathrm{x}$ ambientes (GxE). Essas interações dificultam o trabalho dos melhoristas, que consiste na identificação de genótipos superiores, seja na ocasião da seleção, seja no momento da recomendação de cultivares (Oliveira et al., 2003).

Cada melhorista deve decidir qual deverá ser o ambiente a receber os genótipos selecionados. Essa decisão pode ser tomada ao se conhecer a natureza da interação. A busca de materiais genéticos que, ao ser avaliados nos vários ambientes, não mostrem alterações significativas, é necessária para maior segurança na recomendação de cultivares. 
Métodos estatísticos têm sido propostos para avaliar a estabilidade e a adaptabilidade da cultivar, contornando, em parte, os inconvenientes da interação genótipo x ambiente (Mandel, 1971; Wricke \& Weber, 1986; Crossa, 1990). A estratificação de ambientes também é importante no estudo da interação, principalmente quando se quer verificar se entre os ambientes da rede experimental há padrões de similaridades de resposta das cultivares avaliadas (Lavoranti, 2003). Isso possibilita avaliar o grau de representatividade dos ensaios na faixa de adaptação da cultura, agrupar ambientes em que a interação GxE seja não-significativa, e decidir, seja por problemas técnicos ou por escassez de recursos, sobre o descarte de ambientes (Carneiro, 1998).

Em tais estudos, o agrupamento é realizado com base nos principais efeitos do genótipo e da interação GxE, ou apenas sobre a interação GxE, ou ainda, de acordo com Gauch Junior \& Zobel (1996) e Duarte \& Vencovsky (1999), combinando, num único modelo, um componente aditivo para os efeitos principais (genótipos e ambientes), e um componente multiplicativo, para os efeitos da interação GxE, pela análise Additive Main Effects and Multiplicative Interaction (AMMI).

A análise AMMI decompõe os efeitos da interação de GxE, em uma porção denominada padrão, que possibilita a identificação de fatores ambientais e genotípicos mais diretamente relacionados à interação, e numa outra porção denominada ruído (Oliveira et al., 2003). Essa análise pode ajudar tanto na identificação de genótipos de alta produtividade e amplamente adaptados quanto na realização do chamado zoneamento agronômico, com fins de recomendação regionalizada e de seleção de locais de testes (Gauch Junior \& Zobel, 1996).

O objetivo deste trabalho foi avaliar a estabilidade e a adaptabilidade da cultura do algodoeiro e, simultaneamente, realizar o zoneamento agronômico, pelo estudo da interação genótipos $\mathrm{x}$ ambientes, usando o método da ecovalência $\left(\mathrm{W}_{\mathrm{i}}^{2}\right)$ e o modelo AMMI.

\section{Material e Métodos}

Foram utilizados dados de produtividade do algodão em caroço obtidos nos ensaios regionais de avaliação de cultivares do algodoeiro herbáceo conduzidos no Cerrado do Mato Grosso e Mato Grosso do Sul, na safra 1998/1999, coordenados pela Embrapa Algodão. Nesses ensaios utilizaram-se as linhagens ou cultivares: CNPA ITA 90 (padrão), BRS Antares, BRS ITA 96,
CNPA 94151 (BRS 197), CNPA 94171 (BRS FACUAL), CNPA 94773, CNPA 95743 (FMT Fetagri) e CNPA 95122 (FMT Saturno).

Foram escolhidos 16 locais, de acordo com a diversidade e representatividade dos diferentes ecossistemas do Cerrado, incluindo os Estados de Mato Grosso e Mato Grosso do Sul, nos Municípios: Campo Novo dos Parecis 1 $\left(\mathrm{CNP}_{1}\right)$, Campo Novo dos Parecis $2\left(\mathrm{CNP}_{2}\right)$, Chapadão do Sul (CHAP), Sorriso (SOR), Cáceres (CAC), Nova Mutum (NM), São José dos Quatro Marcos (QM), Ponta Porã (PPO), Primavera do Leste (PRI), Rondonópolis (ROO), Itiquira (ITI), Campo Verde (CV), Sapezal (SAP), Pedra Preta (PPA), Alto Taquari (ALT), e São Lucas do Rio Verde (LRV).

O delineamento utilizado foi o quadrado latino 8x8. As parcelas foram constituídas por quatro linhas de $5 \mathrm{~m}$ de comprimento, com espaçamento de $0,90 \mathrm{~m}$ entre linhas. Utilizaram-se, como bordadura, duas fileiras da cultivar CNPA ITA 90 no início e final de cada bloco. A área total foi $1.976,40 \mathrm{~m}^{2}$, e as duas fileiras centrais foram consideradas a área útil da parcela.

Realizou-se análise de variância individual, quanto ao caráter produtividade do algodão em caroço. Em seguida, foi realizada a análise de variância conjunta, conforme metodologia proposta por Gomes (1990), considerando-se fixos os efeitos de genótipos e de ambientes.

Utilizou-se inicialmente, na avaliação da estabilidade, o método da ecovalência (Wricke \& Weber, 1986), que é um dos procedimentos mais empregados pelos melhoristas europeus, sobretudo pela facilidade de interpretação. Esse método estima o que se denomina ecovalência $\left(\mathrm{W}_{\mathrm{i}}^{2}\right)$, obtida por meio da partição da soma de quadrados da interação GxE e, é fornecida por: $\mathrm{W}_{\mathrm{i}}^{2}=\sum_{\mathrm{j}=1}^{\mathrm{n}}\left(\mathrm{Y}_{\mathrm{ij}}-\overline{\mathrm{Y}}_{\mathrm{i}},-\overline{\mathrm{Y}}_{\mathrm{j}}+\overline{\mathrm{Y}}_{. .}\right)^{2}=\sum_{\mathrm{j}=1}^{\mathrm{n}}\left[(\hat{\mathrm{ge}})_{\mathrm{ij}}\right]^{2}$ em que $\mathrm{W}_{\mathrm{i}}^{2}$ é a ecovalência; $\mathrm{Y}_{\mathrm{ij}}$ é a média da cultivar i no ambiente j; $\bar{Y}_{i}$ é a média da cultivar i; $\bar{Y}_{j}$ é a média do ambiente j; $\mathrm{e}$ $\bar{Y}$.. é a média geral e $\sum_{\mathrm{j}=1}^{\mathrm{n}}\left[(\hat{\mathrm{g} e})_{\mathrm{ij}}\right]$ é a soma de quadrado da interação GxE.

O somatório dos $\mathrm{W}_{\mathrm{i}}^{2}$ corresponde ao valor da soma de quadrados da interação GxE. Dessa forma, é possível calcular a porcentagem da interação $\mathrm{GxE}$ devida a cada genótipo $\left(\mathrm{W}_{\mathrm{i}}^{2} \%\right)$, dada pela equação: $\mathrm{W}_{\mathrm{i}}^{2} \%=\left(\overline{\mathrm{W}}_{\mathrm{i}}^{2} / \sum \overline{\mathrm{W}}_{\mathrm{i}}^{2}\right) 100$. 
Posteriormente, utilizou-se o modelo AMMI para avaliar a adaptabilidade e a estabilidade e se fazer o zoneamento agronômico das linhagens e cultivares (Mandel, 1971). Enquanto o modelo da ecovalência utiliza a soma de quadrados (SQ) da interação na íntegra, no modelo AMMI, essa SQ é desdobrada em componentes relativos a cada eixo da interação da análise de componentes principais (IPCA $A_{k}$ ), descartando o ruído.

O efeito da interação do genótipo i, com o ambiente j, é descrito como a soma de $\mathrm{n}$ parcelas, cada uma resultante da multiplicação de $\lambda_{\mathrm{k}}$, expresso na mesma unidade de $Y_{\mathrm{ij}}$, por efeitos de escala (adimensionais) genotípico $\left(\gamma_{\mathrm{ik}}\right)$ e ambiental $\left(\alpha_{\mathrm{jk}}\right)$. O termo $\lambda_{\mathrm{k}}$ contém informação relativa à variação resultante da interação $G x E$, na k-ésima dessas parcelas. Como numa análise de componentes principais (ACP), essas sucessivas parcelas captarão porções cada vez menores da variação presente na matriz GxE $\left(\lambda_{1}^{2} \geq \lambda_{2}^{2} \geq \ldots \geq \lambda_{\mathrm{p}}^{2}\right)$. Dessa forma, a análise buscará recuperar uma parcela determinada da matriz denominada padrão, descartando-se outra denominada ruído $\left(\rho_{\mathrm{ij}}\right)$. Por isso, o modelo AMMI é visto como procedimento capaz de separar padrão e ruído na análise da $S_{\mathrm{GxE}}$ (Weber et al., 1996). De acordo com esse método, o padrão é a possível lei que governa o fenômeno, e o ruído é a porção presente na interação que é descartada, buscando-se melhorar a capacidade preditiva do modelo, com benefícios diretos à seleção de genótipos (Zobel et al., 1988).

Um dos procedimentos na determinação dos graus de liberdade associados à parcela da $\mathrm{SQ}_{\mathrm{GxE}}$ é obtido pelo teste F, avaliando-se a significância de cada componente em relação ao $\mathrm{QM}_{\text {(erro médio). Isso resulta num quadro de }}$ análise de variância semelhante ao tradicional, com desdobramento da fonte de variação interação (GxE). Dessa forma, o ponto de parada que determinará a seleção dos modelos $\left(\mathrm{AMMI}_{0}, \mathrm{AMMI}_{1}, \ldots \ldots . . . . . \mathrm{AMMI}_{8}\right)$ foi obtido com base na significância do teste $F_{\text {Gollob }}$ para os sucessivos termos da interação, por esse critério ser de fácil aplicação, requerendo uma aritmética trivial (Gollob, 1968).

O efeito multiplicativo da interação será avaliado pela ACP, da soma de quadrados da interação ( $\mathrm{SQ}_{\mathrm{GxE}}$ ), em oito eixos ou componentes principais da interação (IPCA $\mathrm{K}_{\mathrm{k}}$ ), sendo adotado o critério preditivo, por meio do teste $\mathrm{F}_{\mathrm{Gollob}}$, que mostrou ser eficiente para a escolha do modelo AMMI. Os resultados serão também analisados pela representação gráfica em biplot (Gabriel, 1971).
O cálculo dos ângulos vetores baseou-se nas recomendações de Kroonenberg (1997), que salienta que o ângulo entre dois vetores genotípicos ou ambientais pode ser diretamente lido ou medido de um gráfico, podendo ser indicado $\overrightarrow{\mathrm{X}}$ e $\overrightarrow{\mathrm{Y}}$ como $\theta_{\mathrm{xy}}$.

\section{Resultados e Discussão}

Pelos resultados das análises de variância individual e conjunta, houve diferenças significativas, a 1\% de probabilidade, para os efeitos de genótipos (G), ambientes (E) e interação (GxE). O efeito de ambientes foi responsável pela maior parte da variação dos tratamentos seguida pela interação e, por último, pelos genótipos.

A ordem classificatória de estabilidade genotípica para as cultivares e linhagem, pelo método da ecovalência, está indicada na Tabela 1. Em primeiro lugar, destacouse o genótipo CNPA 94773, considerado o mais estável pela menor estimativa de ecovalência $(268,8)$ e, portanto, o que menos contribuiu para os efeitos da interação GxE (3,8\%). Em segundo lugar, na ordem crescente de valores, e decrescente de estabilidade, encontra-se o genótipo CNPA 95743, contribuindo com apenas 4,6\% para os efeitos da interação $\mathrm{GxE}$, seguidos pelo CNPA 95122 (5,5\%), CNPA 94151 (7\%), BRS ITA 96 (9,3\%), BRS Antares (12\%), CNPA 94171 (16,7\%) e, em último lugar, com maior contribuição para a interação, encontra-se a cultivar CNPA ITA 90, contribuindo com $41,1 \%$ para os efeitos da interação, enfatizando sua instabilidade ante as variações ambientais, principalmente devido aos níveis de resistência a doenças fúngicas e viróticas, já que essa cultivar tem sensibilidade comprovada à virose causadora da doença azul (Farias et al., 1999), cuja incidência foi marcante durante a realização dos ensaios.

Tabela 1. Parâmetros de estabilidade para a produtividade de oito genótipos do algodoeiro herbáceo, avaliados em 16 ambientes no Cerrado de Mato Grosso e Mato Grosso do Sul, na safra 1998/1999, com base na metodologia da ecovalência $\left(\hat{\mathrm{W}}_{\mathrm{i}}^{2}\right)$.

\begin{tabular}{lrrcr}
\hline Cultivar/linhagem & $\begin{array}{c}\text { Média } \\
\left(\mathrm{kg} \mathrm{ha}^{-1}\right)\end{array}$ & $\begin{array}{c}\left(\hat{\mathrm{W}}_{\mathrm{i}}^{2}\right) \\
/ 1000\end{array}$ & $\begin{array}{c}\text { Ordem de } \\
\text { estabilidade }\end{array}$ & $\%\left(\hat{\mathrm{W}}_{\mathrm{i}}^{2}\right)$ \\
\hline CNPA ITA 90 & 3.650 & $2.894,3$ & 8 & 41,1 \\
BRS Antares & 3.664 & 848,0 & 6 & 12,0 \\
BRS ITA 96 & 3.771 & 652,4 & 5 & 9,3 \\
CNPA 94151 & 3.907 & 493,3 & 4 & 7,0 \\
CNPA 94171 & 3.961 & $1.178,2$ & 7 & 16,7 \\
CNPA 94773 & 3.789 & 268,8 & 1 & 3,8 \\
CNPA 95743 & 3.884 & 322,4 & 2 & 4,6 \\
CNPA 95122 & 3.893 & 383,9 & 3 & 5,5 \\
\hline
\end{tabular}


Apesar da facilidade de interpretação deste método, não há possibilidade de se saber, por exemplo, se dois genótipos com a mesma ecovalência têm o mesmo padrão de comportamento ao longo dos ambientes. O método também não identifica os pares de genótipos e ambientes que capitalizam de forma positiva os efeitos da interação, em virtude da ausência de análise gráfica. Como esse procedimento não informa sobre interações específicas de genótipos com ambientes (se positivas ou negativas), dificultando a exploração vantajosa dos efeitos da interação, utilizou-se outro modelo (AMMI), que vem ganhando aplicabilidade nos últimos anos.

A porcentagem acumulada explicada pelos dois primeiros eixos da interação, para a estrutura-padrão, pelo modelo AMMI, foi de 79,03\% (Tabela 2). A interpretação dos resultados se torna relativamente simples pelo padrão ser reduzido a duas dimensões (Chaves, 2001), em que se considera apenas a variação contida nos dois primeiros eixos da análise de componentes principais, como suficiente para avaliar a adaptabilidade e a estabilidade dos genótipos e ambientes (Rocha, 2002).

Esse porcentual alcançado está em desacordo com Arias et al. (1996), os quais salientam que esta metodologia, geralmente, apresenta baixos valores porcentuais para explicação dos primeiros componentes principais da interação. No entanto, Zobel et al. (1988), avaliando sete genótipos de soja em 35 ambientes, encontraram um porcentual de $71 \%$ para o primeiro eixo da interação. Por sua vez, Freire Filho et al. (2003) observaram em seus estudos que mesmo com os dois primeiros eixos da interação explicando apenas $61,25 \%$ da variabilidade devida à interação, esse porcentual per-

Tabela 2. Análise de variância conjunta com desdobramento da interação GxE, atribuindo-se graus de liberdade aos componentes da interação (IPCA $A_{k}$ ), conforme o sistema de Gollob (1968). Dados de produtividade de algodão em caroço.

\begin{tabular}{lrcc}
\hline Fonte de variação & $\mathrm{GL}$ & \multicolumn{1}{c}{$\mathrm{QM}$} & \multicolumn{1}{c}{$\mathrm{F}_{\text {Gollob }}$} \\
\hline Genótipo $(\mathrm{G})$ & 7 & 1.468 .948 & $3,13^{* *}$ \\
Ambiente (E) & 15 & 77.073 .630 & $164,33^{* *}$ \\
Interação (GxE) & 105 & 469.010 & $3,44^{* *}$ \\
IPCA $_{1}$ (padrão) & 21 & 1.179 .560 & $8,65^{* *}$ \\
IPCA $_{2}$ & 19 & 271.910 & $1,99^{*}$ \\
Resíduo AMMI & 65 & $38.717^{\text {ns }}$ & $0,28^{\text {ns }}$ \\
\hline Resíduo & 672 & 136.294 & - \\
\hline
\end{tabular}

ns Não-significativo. * e **Significativo a 5 e $1 \%$ de probabilidade pelo teste F de Gollob. mitiu-lhes selecionar genótipos de feijão-caupi que reunissem genes conferindo estabilidade e adaptabilidade, e que, além disso, pudessem ser recomendados para todos os ambientes testados.

Proporções maiores de variabilidade, explicadas pelos dois primeiros eixos da interação, indicam que o gráfico baseado em dois vetores singulares fornecerá boa representação da estrutura-padrão na tabela (Kroonenberg, 1997), aumentando a precisão das inferências das estabilidades de genótipos e de ambientes.

Na Figura 1 está a representação gráfica (biplot) dos genótipos e dos ambientes, resultante da análise pelo modelo $\mathrm{AMMI}_{1}$, para os dados analisados. A abscissa representa os efeitos principais (médias de genótipos e de ambientes), e a ordenada, o primeiro eixo da interação $\left(\right.$ IPCA $\left._{1}\right)$.

Uma propriedade desse tipo de representação gráfica (biplot) é que, calculando-se o produto interno entre um vetor linha e um vetor coluna, épossível identificar a adaptação específica, bem como as interações positivas de GxE, pelo cálculo de seus ângulos vetores genotípicos e ambientais. Esse ângulo informará sobre a correlação

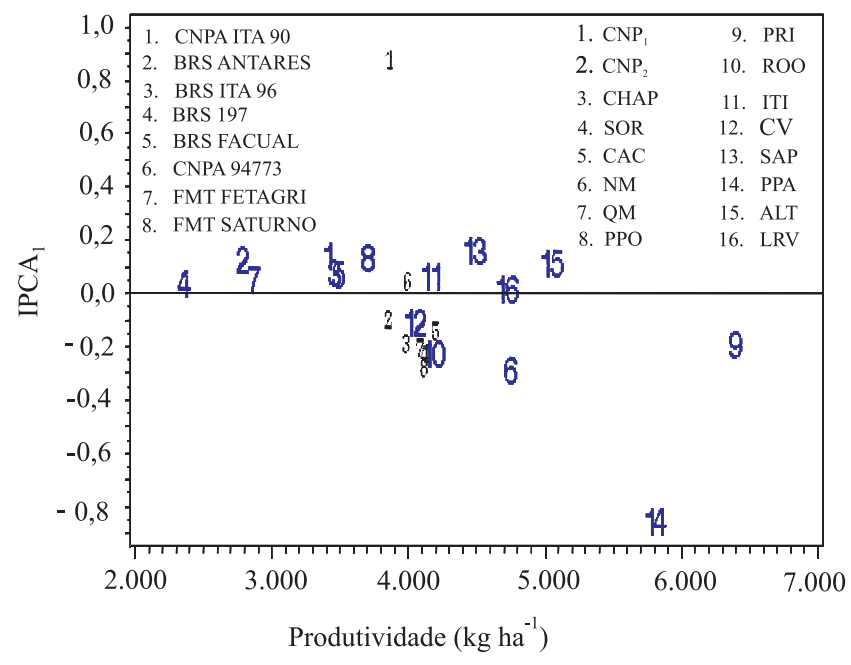

Figuras 1. Biplot $\mathrm{AMMI}_{1}$ para dados de produtividade de oito genótipos do algodoeiro herbáceo (preto) avaliados em 16 ambientes (azul) do Cerrado do Mato Grosso e Mato Grosso do Sul, na safra 1998/1999. CNP $_{1}$ : Campo Novo dos Parecis - local 1; $\mathrm{CNP}_{2}$ : Campo Novo dos Parecis local 2; CHAP: Chapadão do Sul; SOR: Sorriso; CAC: Cáceres; NM: Nova Mutum; QM: São José dos Quatro Marcos; PPO: Ponta Porã; PRI: Primavera do Leste; ROO: Rondonópolis; ITI: Itiquira; CV: Campo Verde; SAP: Sapezal; PPA: Pedra Preta; ALT: Alto Taquari e LRV: São Lucas do Rio Verde. 
linear entre dois vetores e, conseqüentemente, entre as variáveis a eles correlacionadas (Tabela 3 ).

Dessa forma, genótipos com IPCA A $_{1}$ próximo a zero demonstram estabilidade aos ambientes de teste, como o genótipo CNPA 94773, que contribuiu pouco para a interação total, e foi considerado como o mais estável, por esse critério de avaliação, por ele demonstrar adaptabilidade geral e menor variabilidade entre os ambientes (Figura 1). Quanto aos efeitos aditivos principais de genótipos e ambientes, observa-se pouca variabilidade entre genótipos, que se agruparam na coluna em torno da média geral; já para os efeitos de ambientes, houve maior variabilidade (Figura 1).

Assim, combinações de genótipos com ambientes afastados da origem, e próximos entre si, mostram interação positiva, representando adaptações específicas. É o caso do genótipo BRS Antares com os ambi- entes de Ponta Porã, São Lucas do Rio Verde e Alto Taquari, com ângulos respectivos de $24^{\circ}, 25^{\circ}$ e $34^{\circ}$; do genótipo CNPA 94151, com os ambientes Nova Mutum e Campo Verde, cujos ângulos são respectivamente, $24^{\circ} \mathrm{e}$ 25; além do genótipo CNPA 94171, com os ambientes Campo Verde e São José dos Quatro Marcos, com ângulos de $1,39^{\circ}$ e $23,8^{\circ}$, respectivamente (Tabela 3).

Os genótipos mais produtivos foram o CNPA 94171 (3.961 kg ha-1), CNPA 94151 (3.907 kg ha-1), CNPA 95122 (3.893 $\mathrm{kg} \mathrm{ha}^{-1}$ )eCNPA 95743(3.884 $\left.\mathrm{kg} \mathrm{ha}^{-1}\right)$, com produtividades acima da média geral dos ensaios experimentais $\left(3.814,75 \mathrm{~kg} \mathrm{ha}^{-1}\right)$. Esses genótipos são considerados de elevada adaptabilidade (Tabela 4). Por sua vez, o mais estável foi o genótipo CNPA 94773, considerado de ampla adaptabilidade, e, conseqüentemente, elevada estabilidade pelos dois métodos utilizados (ecovalência

Tabela 3. Ângulos vetores genotípicos em relação aos vetores ambientes, obtidos dos produtos internos de cada genótipo, em cada um dos ambientes pesquisados. Dados de produtividade de algodão em caroço.

\begin{tabular}{|c|c|c|c|c|c|c|c|c|}
\hline \multirow[t]{3}{*}{ Ambientes } & \multicolumn{8}{|c|}{ Genótipos } \\
\hline & CNPA & BRS & BRS & CNPA & CNPA & CNPA & CNPA & CNPA \\
\hline & ITA 90 & Antares & ITA 96 & 94151 & 94171 & 94773 & 95743 & 95122 \\
\hline$\overline{\mathrm{CNP}_{1}}$ & 2,4 & 96,9 & 146,5 & 123,2 & 99,2 & 50,0 & 135,0 & 155,9 \\
\hline $\mathrm{CNP}_{2}$ & 42,7 & 141,9 & 168,5 & 78,2 & 54,1 & 95,0 & 179,9 & 159,1 \\
\hline CHAP & 43,4 & 142,7 & 167,8 & 77,4 & 53,4 & 95,7 & 179,2 & 158,4 \\
\hline SOR & 9,43 & 108,7 & 158,3 & 111,4 & 87,4 & 61,8 & 146,8 & 167,7 \\
\hline $\mathrm{CAC}$ & 59,5 & 158,7 & 151,7 & 61,4 & 37,3 & 111,8 & 163,1 & 142,3 \\
\hline NM & 145,0 & 115,7 & 66,1 & 24,2 & 48,2 & 162,6 & 77,5 & 56,7 \\
\hline QM & 73,0 & 172,3 & 138,1 & 47,8 & 23,8 & 125,4 & 149,6 & 128,8 \\
\hline $\mathrm{PPO}$ & 74,8 & 24,5 & 74,1 & 164,4 & 171,6 & 22,4 & 62,6 & 83,5 \\
\hline PRI & 157,3 & 103,4 & 53,8 & 36,5 & 60,5 & 150,3 & 65,2 & 44,4 \\
\hline ROO & 137,9 & 38,7 & 10,9 & 101,2 & 125,3 & 85,6 & 0,5 & 20,3 \\
\hline ITI & 15,3 & 83,9 & 133,5 & 136,2 & 112,1 & 37,0 & 122,1 & 142,9 \\
\hline $\mathrm{CV}$ & 95,4 & 165,3 & 115,8 & 25,4 & 1,4 & 147,8 & 127,2 & 106,4 \\
\hline SAP & 42,8 & 56,5 & 106,0 & 163,6 & 139,6 & 9,5 & 94,6 & 115,4 \\
\hline PPA & 171,1 & 71,8 & 22,2 & 68,1 & 92,1 & 118,7 & 33,7 & 12,9 \\
\hline ALT & 65,0 & 34,3 & 83,8 & 174,1 & 161,8 & 12,7 & 72,4 & 93,2 \\
\hline LRV & 74,2 & 25,1 & 74,6 & 164,9 & 171,0 & 21,8 & 63,2 & 84,0 \\
\hline
\end{tabular}

Tabela 4. Produtividade média $\left(\mathrm{kg} \mathrm{ha}^{-1}\right)$ de oito cultivares do algodoeiro herbáceo, produzido e avaliado em 16 ambientes do Cerrado do Mato Grosso e Mato Grosso do Sul, na safra 1998/1999.

\begin{tabular}{|c|c|c|c|c|c|c|c|c|c|c|c|c|c|c|c|c|c|c|}
\hline \multirow{2}{*}{$\begin{array}{l}\text { Genótipos/ } \\
\text { cultivares }\end{array}$} & \multicolumn{16}{|c|}{ Ambientes $^{(1)}$} & \multirow{2}{*}{$\begin{array}{c}\text { Médias } \\
\left(\mathrm{Y}_{\mathrm{i}}\right)\end{array}$} & \multirow{2}{*}{$\begin{array}{c}\text { Efeito } \\
\text { gen }\left(g_{i}\right)\end{array}$} \\
\hline & $\mathrm{CNP}_{1}$ & $\mathrm{CNP}_{2}$ & CHAP & SOR & $\mathrm{CAC}$ & NM & $\mathrm{QM}$ & $\mathrm{PPO}$ & PRI & $\mathrm{ROO}$ & ITI & $\mathrm{CV}$ & SAP & PPA & ALT & LRV & & \\
\hline CNPA ITA 90 & 3.288 & 2.589 & 3.161 & 1.964 & 3.167 & 3.839 & 2.586 & 3.491 & 5.676 & 3.429 & 3.887 & 3.516 & 4.418 & 4.106 & 4.892 & 4.392 & 3.650 & -165 \\
\hline BRS Antares & 3.041 & 2.299 & 2.683 & 1.828 & 2.749 & 4.003 & 2.184 & 3.513 & 5.780 & 3.961 & 3.827 & 3.286 & 4.194 & 5.832 & 4.994 & 4.449 & 3.664 & -155 \\
\hline BRS ITA 96 & 2.754 & 2.239 & 3.158 & 2.155 & 3.184 & 4.516 & 2.527 & 3.660 & 5.655 & 4.093 & 3.671 & 3.866 & 3.936 & 5.680 & 4.683 & 4.553 & 3.771 & -44 \\
\hline CNPA 94151 & 3.203 & 2.480 & 3.339 & 1.995 & 3.457 & 4.733 & 2.846 & 2.996 & 6.325 & 3.894 & 3.885 & 3.891 & 4.263 & 5.901 & 4.717 & 4.595 & 3.907 & 92 \\
\hline CNPA 94171 & 3.282 & 2.855 & 3.234 & 2.261 & 3.501 & 4.688 & 3.107 & 2.968 & 6.224 & 3.913 & 4.125 & 4.517 & 4.191 & 5.644 & 4.551 & 4.307 & 3.960 & 145 \\
\hline CNPA 94773 & 2.836 & 2.441 & 3.166 & 2.151 & 3.272 & 4.400 & 2.395 & 3.569 & 6.160 & 3.826 & 3.870 & 3.673 & 4.078 & 5.420 & 4.780 & 4.584 & 3.789 & -26 \\
\hline CNPA 95743 & 3.252 & 2.529 & 3.160 & 1.984 & 3.128 & 4.601 & 2.381 & 3.509 & 6.413 & 4.079 & 3.880 & 3.703 & 4.392 & 5.926 & 4.925 & 4.288 & 3.884 & 69 \\
\hline CNPA 95122 & 3.151 & 2.400 & 3.268 & 2.052 & 2.978 & 4.694 & 2.483 & 3.415 & 6.427 & 4.154 & 4.032 & 3.850 & 4.320 & 5.831 & 4.753 & 4.483 & 3.893 & 78 \\
\hline$\overline{\text { Média }\left(Y_{\mathrm{j}}\right)}$ & 3.100 & 2.479 & 3.146 & 2.049 & 3.179 & 4.434 & 2.564 & 3.390 & 6.082 & 3.919 & 3.897 & 3.788 & 4.224 & 5.542 & 4.787 & 4.456 & $3.815,4$ & - \\
\hline Ef. de $a m b\left(a_{i}\right)$ & -715 & -1.340 & -669 & -1.760 & -635 & 619 & -1.251 & -420 & 2.267 & 104 & 82 & -27 & 409 & 1.727 & 972 & 641 & - & - \\
\hline $\mathrm{CV}(\%)$ & 9.69 & 12.58 & 10.51 & 10.80 & 10.16 & 9.16 & 10.91 & 11.28 & 8.58 & 10.32 & 8.74 & 8.1 & 7.2 & 8.95 & 10.08 & 7.89 & & \\
\hline
\end{tabular}

${ }^{(1)} \mathrm{CNP}_{1}$ : Campo Novo dos Parecis; $\mathrm{CNP}_{2}$ : Campo Novo dos Parecis; CHAP: Chapadão do Sul; SOR: Sorriso; CAC: Cáceres; NM: Nova Mutum; QM: São José dos Quatro Marcos; PPO: Ponta Porã; PRI: Primavera do Leste; ROO: Rondonópolis; ITI: Itiquira; CV: Campo Verde; SAP: Sapezal; PPA: Pedra Preta; ALT: Alto Taquari e LRV: Lucas do Rio Verde. 
e AMMI), esse genótipo pode ser recomendado para todos os locais da pesquisa.

Os ambientes mais favoráveis a uma boa produtividade foram Primavera do Leste, Pedra Preta, Alto Taquari, Lucas do Rio Verde, Nova Mutum, Sapezal, Rondonópolis, Itiquira e Campo Verde, cujos escores de genótipos e de ambientes foram plotados, adequandose ao modelo $\mathrm{AMMI}_{2}$.

Ao se considerar a produtividade média de todos os genótipos nos ambientes Campo Novo dos Parecis $_{1}$ (3.100 kg ha-1), Campo Novo dos Parecis 2 (2.479 $\mathrm{kg} \mathrm{ha}^{-1}$ ), Sorriso (2.048 kg ha-1) e São José dos Quatro Marcos (2.563 $\mathrm{kg} \mathrm{ha}^{-1}$ ), verifica-se que foram os mais desfavoráveis ao desempenho do algodoeiro (Tabela 4).

O maior rendimento de algodão em caroço, nesses locais, foi alcançado pelo genótipo CNPA 94171, com valores de $3.238 \mathrm{~kg} \mathrm{ha}^{-1}\left(\mathrm{CNP}_{1}\right), 2.794 \mathrm{~kg} \mathrm{ha}^{-1}\left(\mathrm{CNP}_{2}\right)$, $2.225 \mathrm{~kg} \mathrm{ha}^{-1}$ (SOR) e $3.090 \mathrm{~kg} \mathrm{ha}^{-1}(\mathrm{QM})$; esse genótipo também superou os demais em produtividade nos ambientes de desempenho médio como Chapadão do Sul (3.146 kg ha-1), Cáceres (3.179 kg ha-1) e Campo Verde (3.788 $\mathrm{kg} \mathrm{ha}^{-1}$ ), com produtividades médias de $3.425 \mathrm{~kg} \mathrm{ha}^{-1}, 3.553 \mathrm{~kg} \mathrm{ha}^{-1} \mathrm{e} 4.462 \mathrm{~kg} \mathrm{ha}^{-1}$, respectivamente. Nos ambientes favoráveis à boa produtividade do algodoeiro, como Itiquira, Nova Mutum e Primavera do Leste, esse genótipo alcançou produtividade média, de $4.026 \mathrm{~kg} \mathrm{ha}^{-1}, 4.827 \mathrm{~kg} \mathrm{ha}^{-1}$ e $6.348 \mathrm{~kg} \mathrm{ha}^{-1}$, respectivamente, e foi considerado também de elevada adaptabilidade, além de estabilidade geral, tanto em ambientes desfavoráveis quanto favoráveis, por isso foi lançado, na safra seguinte, como cultivar, pela Embrapa, com a denominação BRS FACUAL.

No biplot $\mathrm{AMMI}_{2}$ (Figura 2), são visualizados apenas efeitos multiplicativos da interação GxE, contidos nos dois primeiros componentes principais da interação (CPI) e dispersos em ambas as ordenadas do gráfico. Genótipos e ambientes estáveis são aqueles cujos pontos situamse próximo à origem, ou seja, com escores praticamente nulos para os dois eixos da interação (Duarte \& Vencovsky, 1999). O valor estimado da interação, para uma combinação específica de genótipos com ambientes, é obtido multiplicando-se cada eixo da interação por suas coordenadas IPCA respectivas, somando-se os resultados dos dois eixos. Assim, combinações de genótipos com ambientes afastados da origem, e próximos entre si, mostram interação positiva, representando adaptações específicas (Figura 2).
Da mesma forma, verificou-se interação específica positiva dos genótipos BRS ITA 96, CNPA 95743 e CNPA 95122 com os ambientes Rondonópolis e Pedra Preta; CNPA 94151, com os ambientes Nova Mutum, Primavera do Leste e Campo Verde; CNPA 94171, com os ambientes São José dos Quatro Marcos e Campo Verde; CNPA 94773 com os ambientes Ponta Porã, Sapezal, Alto Taquari e São Lucas do Rio Verde; e por último, do genótipo BRS Antares, com os ambientes Ponta Porã, Alto Taquari e São Lucas do Rio Verde (Figura 2).

Um pouco mais distante da origem, em uma porção mais intermediária, encontram-se os genótipos BRS ITA 96, CNPA 95743, CNPA 95122 e CNPA 94151, os quais podem ser considerados estáveis; mais distanciados da origem, porém, com valores baixos de escores apenas no primeiro eixo da interação, encontram-se os genótipos BRS Antares e CNPA 94171, também considerados estáveis. O ambiente de Primavera do Leste, por estar posicionado próximo à origem, pode ser considerado o mais representativo das condições ambientais, com relação aos fatores importantes para a interação. Este tipo de informação, de acordo com Chaves (2001), pode ser importante na escolha-chave de ambientes para avaliação de genótipos em futuros programas de melhoramento.

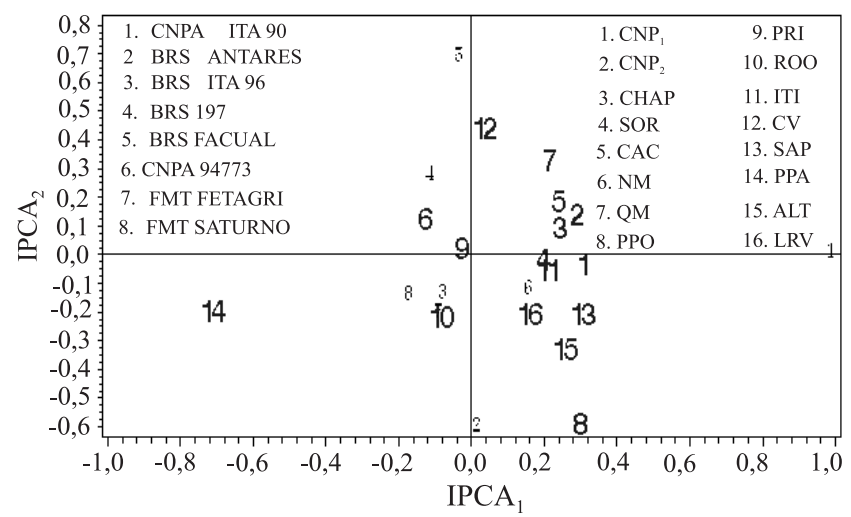

Figura 2. Biplot $\mathrm{AMMI}_{2}$ considerando os dois primeiros eixos da análise de componentes principais para dados de oito genótipos do algodoeiro herbáceo (preto), avaliados em 16 ambientes (azul) do Cerrado do Mato Grosso e Mato Grosso do Sul, na safra 1998/1999. CNP 1 : Campo Novo dos Parecis - local 1; $\mathrm{CNP}_{2}$ : Campo Novo dos Parecis - local 2; CHAP: Chapadão do Sul; SOR: Sorriso; CAC: Cáceres; NM: Nova Mutum; QM: São José dos Quatro Marcos; PPO: Ponta Porã; PRI: Primavera do Leste; ROO: Rondonópolis; ITI: Itiquira; CV: Campo Verde; SAP: Sapezal; PPA: Pedra Preta; ALT: Alto Taquari e LRV: São Lucas do Rio Verde. 


\section{Conclusões}

1. A análise AMMI, pelo modelo selecionado (biplot $\mathrm{AMMI}_{2}$ ), é eficiente e explica melhor a estabilidade e a adaptabilidade dos genótipos e dos ambientes.

2. Os genótipos CNPA 94773 e FMT Fetagri são os mais estáveis; o genótipo BRS FACUAL apresenta elevada produtividade e é o mais adaptável.

3. Existem grupos de adaptabilidade específica e interação positiva entre os genótipos e ambientes avaliados.

\section{Referências}

ARIAS, E.R.A.; RAMALHO, M.A.P.; FERREIRA, D.F. Adaptabilidade e estabilidade de cultivares de milho avaliados no Estado de Mato Grosso do Sul. Ciência e Agrotecnologia, v.20, p.415-420, 1996.

CARNEIRO, P.C.S. Novas metodologias de análise da adaptabilidade e estabilidade de comportamento. 1998. 128p. Tese (Doutorado) - Universidade Federal de Viçosa, Viçosa.

CHAVES, L.J. Interação genótipos com ambientes. In: NASS, L.L.; VALOIS, A.C.C.; MELO, I.S. de; VALADARES-INGLIS, M.C. (Ed.). Recursos genéticos e melhoramento: plantas. Rondonópolis: Fundação MT, 2001. p.674-713.

CROSSA, J. Statistical analysis of multilocation trials. Advances in Agronomy, v.44, p.55-85, 1990.

DUARTE, J.B.; VENCOVSKY, R. Interação genótipo x ambiente: uma introdução à análise "AMMI”. Ribeirão Preto: Sociedade Brasileira de Genética, 1999. 60p. (Série monografias, 9).

FARIAS, F.J.C.; FREIRE, E.C.; AGUIAR, P.H. Desempenho dos cultivares e linhagens do algodoeiro herbáceo no Cerrado do Mato Grosso. In: CONGRESSO BRASILEIRO DE ALGODÃO, 2., 1999, Ribeirão Preto. Anais. Campina Grande: Embrapa-CNPA, 1999. p.575-577.

FREIRE FILHO, F.R.; RIBEIRO, V.Q.; ROCHA, M.M.; LOPES, A.C.A. Adaptabilidade e estabilidade da produtividade de grãos de genótipos de caupi enramador de tegumento mulato. Pesquisa Agropecuária Brasileira, v.38, p.591-598, 2003.
GABRIEL, K.R. The biplot graphic display of matrices with application to principal component analysis. Biometrika, v.58, p.453-467, 1971.

GAUCH JUNIOR, H.G.; ZOBEL, R.W. AMMI analysis of yield trails. In: KANG, M.S.; GAUCH JUNIOR, H.G. (Ed.). Genotype by environment interaction. Boca Raton: CRC Press, 1996. p.85122.

GOLLOB, H.F. A statistical model which combines features of factor analytic and analysis of variance techniques. Psychometrika, v.33, p.73-115, 1968.

GOMES, L.S. Interação genótipo $\mathbf{x}$ época de plantio em milho (Zea mays L.) em dois locais do oeste do Paraná. 1990. 148p. Dissertação (Mestrado) - Escola Superior de Agricultura Luiz de Queiroz, Piracicaba.

KROONENBERG, P.M. Introduction to biplots for GxE tables. Brisbane, Austrália: The University of Queensland Research Report, 1997. Não paginado. (University of Queensland. Research report, 51).

LAVORANTI, O.J. Estabilidade e adaptabilidade fenotípica através da reamostragem "bootstrap” no modelo AMMI. 2003. 166p. Tese (Doutorado) - Escola Superior de Agricultura Luiz de Queiroz, Piracicaba.

MANDEL, J. A new analysis of variance model for non-additive data. Technometrics, v.13, p.1-18, 1971.

OLIVEIRA, A.B.; DUARTE, J.B.; PINHEIRO, J.B. Emprego da análise AMMI na avaliação da estabilidade produtiva em soja. Pesquisa Agropecuária Brasileira, v.26, p.357-364, 2003.

ROCHA, M.M. Seleção de linhagens experimentais de soja para adaptabilidade e estabilidade fenotípica. 2002. 174p. Tese (Doutorado) - Escola Superior de Agricultura Luiz de Queiroz, Piracicaba.

WEBER, W.E.; WRICKE, G.; WESTERMANN, T. Selection of genotypes and prediction of performance by analyzing genotypeenvironment interactions. In: KANG, M.S.; GAUCH JUNIOR, H.G. (Ed.). Genotype by environment interaction. Boca Raton: CRC Press, 1996. p.353-371.

WRICKE, G.; WEBER, W.E. Quantitative genetics and selection in plant breeding. New York: W. de Gruyter, 1986. 406p.

ZOBEL, R.W.; WRIGHT, M.J.; GAUCH JUNIOR, H.G. Statistical analysis of a yield trial. Agronomy Journal, v.80, p.388-393, 1988. 